OPEN ACCESS

Edited by:

Kirsi Laitinen,

University of Turku, Finland

Reviewed by:

Federica Fogacci,

University of Bologna, Italy

Cain Craig Truman Clark,

Coventry University, United Kingdom

*Correspondence:

Gui-ju Sun

gjsun@seu.edu.cn

Specialty section: This article was submitted to

Nutrition and Metabolism,

a section of the journa

Frontiers in Nutrition

Received: 27 August 2021 Accepted: 31 October 2021 Published: 01 December 2021

Citation:

Xia J-y, Yu J-h, Xu D-f, Yang C, Xia H and Sun G-j (2021) The Effects of Peanuts and Tree Nuts on Lipid Profile

in Type 2 Diabetic Patients: A Systematic Review and Meta-Analysis of Randomized, Controlled-Feeding

\section{The Effects of Peanuts and Tree Nuts on Lipid Profile in Type 2 Diabetic Patients: A Systematic Review and Meta-Analysis of Randomized, Controlled-Feeding Clinical Studies}

\author{
Jia-yue Xia ${ }^{1,2}$, Jun-hui $Y u^{1,2}$, Deng-feng Xu ${ }^{1,2}$, Chao Yang ${ }^{1,2}$, Hui Xia ${ }^{1,2}$ and Gui-ju Sun ${ }^{1,2 *}$ \\ ${ }^{1}$ Key Laboratory of Environmental Medicine and Engineering of Ministry of Education, School of Public Health, Southeast \\ University, Nanjing, China, ${ }^{2}$ Department of Nutrition and Food Hygiene, School of Public Health, Southeast University, \\ Nanjing, China
}

Background: Type 2 diabetes mellitus was found to be associated with metabolic disorders, particularly abnormal glucose and lipid metabolism. Dietary food choices may have profound effects on blood lipids. The primary objective of this study was to examine the effects of peanuts and tree nuts intake on lipid profile in patients with type 2 diabetes.

Methods: According to preferred reporting items for systematic reviews and meta-analysis guidelines, we performed a systematic search of randomized controlled clinical trials and systematic reviews published in PubMed, Web of Science, Embase, Scopus, and Cochrane library, from inception through June 2021. Studies in populations with type 2 diabetes, which compare nuts or peanuts to a controlled-diet group were included. We used the mean difference with $95 \% \mathrm{Cls}$ to present estimates for continuous outcomes from individual studies. In addition, we used the GRADEpro tool to evaluate the overall quality of evidence.

Results: Sixteen studies involving 1,041 participants were eligible for this review. The results showed that peanuts and tree nuts supplementation did not induce significant changes in low-density lipoprotein-cholesterol (LDL-C) (mean difference $=-0.11$; 95\% Cl: $-0.25-0.03, p=0.117$ ) and high-density lipoprotein-cholesterol (HDL-C) (mean difference $=0.01 ; 95 \% \mathrm{Cl}:-0.01-0.04, p=0.400$ ) in patients with type 2 diabetics. In addition, we found that peanuts and tree nuts intake may cause a significantly reduction in total cholesterol (TC) (mean difference $=-0.14 ; 95 \% \mathrm{Cl}$ : $-0.26--0.02, p=0.024$ ) and triglyceride (TG) (mean difference $=-0.10 ; 95 \% \mathrm{Cl}$ : $-0.17--0.02, p=0.010$ ). In the subgroup analysis, a significantly greater reduction in TC was observed in studies which duration was $<12$ weeks (mean difference $=-0.22 ; 95 \% \mathrm{Cl}$ : $-0.37--0.08, p=$ 0.002). The quality of the body of evidence was "moderate" for TC and TG, the quality of evidence for LDL-C and HDL-C were "low." 


\begin{abstract}
Conclusion: Our findings suggest that consuming peanuts and tree nuts might be beneficial to lower TC concentration and TG concentration in type 2 diabetics subjects. Furthermore, peanuts and tree nuts supplementation could be considered as a part of a healthy lifestyle in the management of blood lipids in patients with type 2 diabetes. Given some limits observed in the current studies, more well-designed trials are still needed.
\end{abstract}

Keywords: nuts, peanut, cholesterol, blood lipid, type 2 diabetes mellitus, meta-analysis

\section{INTRODUCTION}

Type 2 diabetes mellitus (T2DM), as the most common progressive metabolic disease, is primarily characterized by insulin resistance, a relative deficiency of insulin, and high blood glucose in specific organs and organisms (1). In the United States, the number of patients with diabetes reached $\sim 23.6$ million, and only 17.9 million of them have been diagnosed according to the National Diabetes Statistics (2). T2DM is implicated in disorders of glucose and lipid metabolism (3). Patients with diabetes seem to primarily focus on blood glucose levels and may consider lipid control as less important $(4,5)$. Dyslipidemia in association with type 2 diabetes, which leads to a high rate of severe cardiovascular diseases, is the major cause of morbidity and mortality (6). Thus, intensive management of lipid concentrations to lower the risk of complications of disease progression is imperative.

Nuts (mainly refer to tree nuts and peanuts), belong to high-density nutritious foods, are rich in beneficial nutrients such as unsaturated fatty acids, fiber, lignans, vitamins, minerals, and other biologically active ingredients (7). The most common and popular edible nuts worldwide include walnuts, almonds, hazelnuts, and pistachios. In addition, it also included macadamias, pine nuts, cashews, pecans, and Brazil nuts. Definition of nuts, from a consumer perspective, also contains peanuts which belong to peanuts or beans in botanical terms, but are largely included in the nut food group. In addition, the nutritional content of peanuts is similar to that of tree nuts $(8,9)$. Nowadays tree nuts, integrated in the Mediterranean Diet Pyramid, are regarded as essential parts of a healthy diet (10).

Over the past years, a large body of evidence has accumulated regarding the relationship between peanuts and tree nuts consumption and health outcomes from both epidemiological and controlled dietary trials (11-13). Studies from epidemiology certainly indicated that mature persons more than 65 years old, supplementing three or more servings of nuts every week, had a lower risk of obesity, diabetes, and metabolic syndrome (14). A meta-analysis included 13 clinical trials revealed that the walnut diets can significantly reduce the low-density lipoproteincholesterol (LDL-C) concentration compared with the control diet. Walnut diets or control diets could not induce significant changes in high-density lipoprotein-cholesterol (HDL-C) and triglycerides (TGs) (15). However, a meta-analysis in 2015 (16) revealed that supplementation of tree nuts may have a significant reduction effect on total cholesterol (TC), LDL-C, and TGs concentration. In addition, Mejia et al. (17) concluded that tree nuts could benefit the metabolic syndrome through a modest decrease in TGs. The conclusions of these studies or reviews, obviously, remain controversial. Although the effects of different types of nuts have been studied in different populations such as hyperlipidemic/hypercholesterolemic population (18, $19)$, healthy subjects $(20,21)$, and population with type 2 diabetes (22-24), and some systematic review and meta-analysis have been conducted to explore the effects of different types of nuts consumption on lipid level, there have been relatively few metaanalyses that have specifically investigated the effects of peanuts and total or different types of tree nuts on lipid profile in the diabetic population, especially in patients with T2DM. Therefore, it is necessary to analyze and determine the effect of peanuts and tree nuts consumption on blood lipids in subjects with type 2 diabetes, and this is the novelty of this study.

To provide better evidence-based guidance on the role of peanuts and tree nuts on lipid profile in type 2 diabetic subjects, we conducted a systematic review and meta-analysis of randomized controlled-feeding dietary clinical trials to assess the effects of peanuts and tree nuts (include walnuts, pistachios, macadamia nuts, pecans, cashews, almonds, hazelnuts, pine nuts, and Brazil nuts) on lipid profile (LDL-C, HDL-C, TC, and TG) in individuals with type 2 diabetes.

\section{MATERIALS AND METHODS}

\section{Literature Search Strategy}

We designed and conducted the study according to the guidelines of the 2020 preferred reporting items for systematic reviews and meta-analysis (PRISMA) statement (25). The search was performed in PubMed, Web of Science, Embase, Scopus, and Cochrane library without language restrictions for relevant literature from inception to June 30, 2021. The search used the terms ("Nuts" OR "Tree nuts" OR "pistachios" OR "pine nuts" OR "brazil nuts" OR "cashews" OR "hazelnuts" OR "almonds" OR "walnuts" OR "pecans" OR "macadamia nuts" OR "peanuts") and (“Cholesterol, HDL” OR "Cholesterol, LDL” OR "Triglycerides” OR "serum lipids" OR "triglyceride” OR "low density lipoprotein" OR "high density lipoprotein" OR “TG” OR "TC" OR "lipid profile" OR "serum lipid") and ("Type 2 diabetes" OR "diabetes mellitus, type II" OR "type 2 diabetes mellitus" OR "type 2 diabetes" OR “Diabetes, Type 2") and ("randomized controlled trial" OR "randomized controlled trial" OR "controlled clinical trial” OR "clinical trial, randomized” OR "randomized, trial" OR "randomized" OR "intervention" OR "controlled trial" OR "random" OR "placebo"). If the references of the included kinds of literature meet the requirements, they will be further screened to identify potentially relevant studies. Two reviewers (JYX and JHY) independently conducted the data 
extraction, and any disagreements in terms of literature research were resolved through rigorous discussion or professional consultation. The details of the search strategy are shown in Supplementary Data 2.

\section{Study Selection}

Original studies that met the following inclusion criteria were selected in the systematic reviews and meta-analysis: (a) randomized controlled trials which compare nuts or peanuts to a controlled-diet group; (b) the study population was limited only to the patients diagnosed with T2DM. Accordingly, the following exclusion criteria were also used: (a) studies that clearly did not fulfill the aforementioned inclusion criteria; (b) animal experiments, case reports, editorials, conference papers, and reviews.

\section{Data Extraction}

Independent extraction of data from eligible articles was performed by two authors according to a specially designed data collection form. Data collection items covered the name of the first author, year of publication, country, mean participant age (year), and the number of subjects: intervention group $(n)$ and control group $(n)$, feeding control, study design, nut type, nuts dose $(\mathrm{g} / \mathrm{d})$, and follow-up period. Furthermore, SD and mean net change of LDL-cholesterol ( $\mathrm{mmol} / \mathrm{l})$, HDL-cholesterol $(\mathrm{mmol} / \mathrm{l}), \mathrm{TC}(\mathrm{mmol} / \mathrm{l})$, and TG $(\mathrm{mmol} / \mathrm{l})$ in each study were extracted.

\section{Quality Assessment}

Literature quality evaluation of included studies was performed according to the Cochrane collaboration risk-of-bias tool (26), and the assessment tool assesses seven domains: random sequence generation, allocation concealment, blinding of participants and personnel, blinding of outcome assessment, incomplete outcome data, selective outcome reporting, and other bias. Based on the recommendations of the Cochrane Handbook, each item was scored as "high risk of bias," "low risk of bias," or "unclear risk" for all selected studies. Any disagreements between the authors were resolved through rigorous discussion.

\section{Quantitative Data Synthesis}

Statistical analyses were conducted using Stata SE 15.0 (Stata Corporation, TX, USA) and RStudio v 4.0.3 (RStudio Inc., MA, USA). The package "meta" (version 4.19-0), and "ggplot2" (version 3.3.5) in RStudio were used in combination with Stata SE to perform all analyses. Original studies did not provide effective data directly, the mean net change of relevant indexes was calculated by subtracting mean change (end value minus baseline value). The last end value was used if there were many endpoints. For parallel-controlled studies, we calculated the mean difference (MD) in the effect by using the change from baseline in the control group and the change from baseline in the intervention group. For cross-over trials, the blood lipid value at the end of the control phase and the blood lipid value at the end of the intervention phase are extracted to compute the $\mathrm{MD}$ in the effect. The net change of SD was calculated based on the formula if studies did not provide the net change of SD.
The formula was used as follows: $\mathrm{SD}_{\text {net change }}=$ $\sqrt{\mathrm{SD}(\text { baseline })^{2}+\mathrm{SD}(\text { end point })^{2}-2 \mathrm{R} \times \mathrm{SD}(\text { baseline }) \times \mathrm{SD}(\text { end point })}$. Where necessary, a correlation coefficient $R$ was used and we calculated it according to the following formula from the Cochrane handbook: $\mathrm{R}=\frac{\left[\left(\mathrm{SD}(\text { baseline })^{2}+\mathrm{SD}(\text { end point })^{2}-\mathrm{SD}(\text { change })^{2}\right)\right]}{\left[2^{*} \mathrm{SD}(\text { baeline }) * \operatorname{SD}(\text { end point })\right]}$. We assumed a correlation coefficient $R=0.5$ according to Higgins et al. (27) and used it to calculate the SD of difference in crossover trials when the study did not provide or have sufficient data to calculate the correlation coefficient. We included trials in which tree nuts and peanuts were directly supplemented or added to the diet. Also, a weighted effect size was used for trials involving multiple interventions in a single study. Paired analyses were performed for all multiple intervention studies (28). We had converted the units from $\mathrm{mg} / \mathrm{dl}$ to $\mathrm{mmol} / \mathrm{l}$ for the meta-analysis. For TC, LDL-C, and HDL-C, $1 \mathrm{mg} / \mathrm{dl}$ was converted to $0.0259 \mathrm{mmol} / \mathrm{l}$; for $\mathrm{TG}, 1 \mathrm{mg} / \mathrm{dl}$ was converted to $0.0113 \mathrm{mmol} / \mathrm{l}$. Statistical significance was defined as two-sided $p<0.05$. The extraction of data from the included studies was combined using the generic inverse variance method and a fixed- and random-effects model depending on the degree of heterogeneity. Heterogeneity was assessed through the $I^{2}$ metrics and chi-squared statistics, either $I^{2}>50 \%$ or $p$-value of $\chi^{2}$ test $<0.10$ was deemed as statistically significant heterogeneity. We used the MD with $95 \%$ CIs to present estimates for continuous outcomes from individual studies. Sources of heterogeneity were explored using sensitivity and subgroup analyses. To determine whether a single study has an undue influence on the overall results, sensitivity analyses were performed by excluding the studies sequentially and repeating the meta-analysis. Sensitivity analyses were also by using the random-effects model with Hartung-Knapp-Sidik-Jonkman adjustment or using correlation coefficient of $0.25,0.5$, and 0.75 for cross-over studies to ascertain whether the overall results were robust to the use of different derived correlation coefficients. Also, we conducted subgroup analyses by prespecified covariates, namely, nut type, nut dose $(\mathrm{g} / \mathrm{d})$, the mean age of participants, study design, duration of trials, controlled diets, and tested these covariates for significance by meta-regression.

\section{Publication Bias}

We assessed the asymmetry of the funnel plot visually, used Begg's rank correlation test and Egger's linear regression test to explore any potential publication bias. Also, a "trim and fill" analysis was used to further observe the stability of results if there was any asymmetry in the funnel plot (29).

\section{Certainty of Evidence}

We used the GRADEpro Guideline Development Tool (www. gradepro.org) to rate the quality of evidence and strength of recommendations for each outcome. Quality of a body of evidence involves consideration of the within-study risk of bias (methodological quality), directness of evidence, heterogeneity, the precision of effect estimates, and risk of publication bias. The GRADE system offers four categories of the quality of the evidence (high; moderate; low; and very low) (30). 


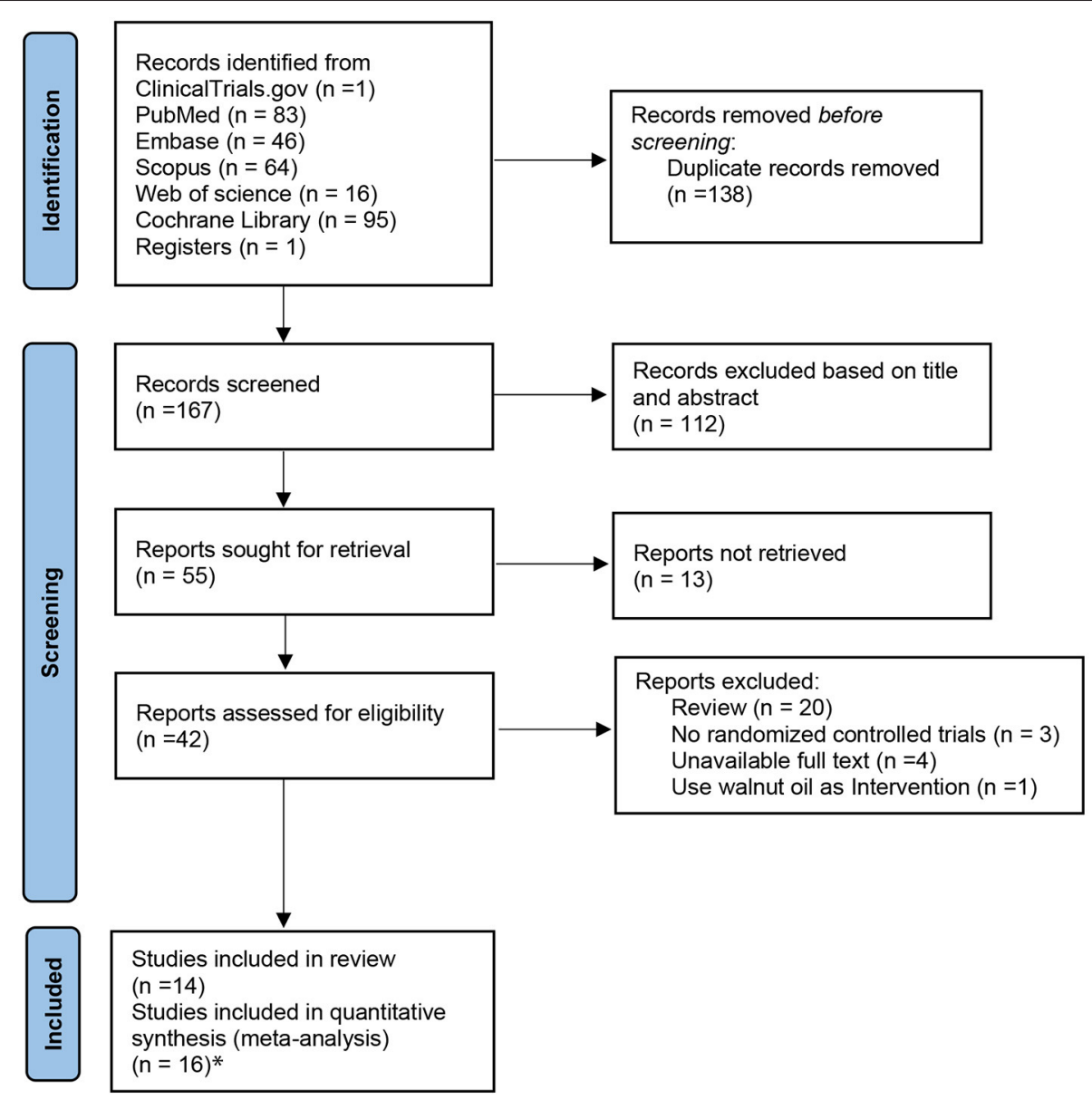

FIGURE 1 | The PRISMA flow diagram of selected trials. Asterisk represents that studies by Jenkins et al. and Lovejoy et al. were each divided into two trials. PRISMA, preferred reporting items for systematic reviews and meta-analysis.

\section{RESULTS}

\section{Study Identification and Selection}

A PRISMA flow diagram of the included trials is shown in Figure 1. A total of 305 articles were identified with the preset search strategy from five electronic databases and one website (ClinicalTrials.gov), of which 250 articles were removed based on duplicate articles, titles, and abstracts. Then, 55 full-text trials were retrieved and only 42 trials were included to be assessed for eligibility; of those, 28 studies were excluded for the following reasons: review articles $(n=20)$, full articles are unavailable $(n=4)$, non-randomized controlled trials $(n$ $=3$ ), and using walnut oil as intervention $(n=1)$. Finally, two of the 14 included studies were divided into two trials, respectively. The study by Lovejoy et al. was divided since it included high almond with high fat and high almond with low fat (31). Jenkins administered two doses of nuts, namely, full-nut dose (mean intake $73 \mathrm{~g} /$ day) and half-nut dose (32). Therefore, 16 trials with sufficient data were eligible for inclusion in the final analysis.

\section{Study Characteristics}

The main characteristics of the included studies are summarized in Table 1 and Supplementary Data 3. These studies were published between 2002 and 2019. Four of them were performed in Asian countries [Taiwan (China) $(33,34)$ and Iran $(22,35)]$, and the other 12 studies were from the United States (31, 3640), Canada (41), Australia (42, 43), and India (44). Nine studies $(22,35,38,39,41-44)$ have a parallel design, while seven $(31,33$, $34,36,37,45)$ have a cross-over design. The sample size ranged from 19 to 300 for a total number of 1,041. The mean age of participants was between 50.1 and 66 years of age. The follow-up period of included studies ranges from 2 weeks to 12 months.

\section{Risk of Bias Assessment}

The risk of bias assessments is shown in Figure 2. Most studies were rated as at low risk of bias. In addition, the risk of random sequence generation in the majority of included trials was unclear because there were insufficient information to generate a risk rating. 
TABLE 1 | Characteristics of included 16 trials in the systematic review and meta-analysis (mean values and standard deviations)

\begin{tabular}{|c|c|c|c|c|c|c|c|c|c|c|}
\hline References & Year & No. of patients & Study region & Mean age, $y(S D)$ & Female (\%) & Comparison group* & Study design & Nut type & Nut dose, $\mathrm{g} / \mathrm{d}$ & Follow-up \\
\hline Lovejoy et al.-HF (31) & 2002 & 30 & USA & $53.8(10.4)$ & $17(50 \%)$ & High-fat control (37\% total fat) & Crossover & Almond & $57-113 \mathrm{~g}$ & $6 w$ \\
\hline Lovejoy et al.-LF (31) & 2002 & 30 & USA & $53.8(10.4)$ & 17 (50\%) & Low-fat control ( $25 \%$ total fat) & Crossover & Almond & $57-113 \mathrm{~g}$ & $6 w$ \\
\hline Tapsell et al. (42) & 2004 & 58 & Australia & $59.3(8.1)$ & $18(31 \%)$ & Low-fat control (low fat, $<30 \%$ fat)Parallel & Walnuts & $30 \mathrm{~g}$ & $6 m$ & \\
\hline Tapsell et al. (43) & 2009 & 50 & Australia & $54(8.7)$ & unclear & $\begin{array}{l}\text { Normal fat ratio control (30\% fat, } 20 \% \\
\text { protein, } 50 \% \text { carbohydrate) }\end{array}$ & Parallel & Walnuts & $30 \mathrm{~g}$ & $12 m$ \\
\hline Ma et al. (36) & 2010 & 24 & USA & $58.1(9.2)$ & $14(58 \%)$ & Self-selected casual diet & Crossover & Walnuts & $56 \mathrm{~g}$ & $8 w$ \\
\hline Li et al. (33) & 2011 & 22 & Taiwan (China) & $58(2)$ & $11(55 \%)$ & $\begin{array}{l}\text { Low-fat control (27\% fat, } 17 \% \text { protein, } 56 \% \\
\text { carbohydrate) }\end{array}$ & Crossover & Almonds & $56 \mathrm{~g}$ & $12 \mathrm{w}$ \\
\hline Jenkins et al.-FD (32) & 2018 & 117 & Canada & $62(9.4)$ & $27(23 \%)$ & Low-fat control (Muffins, total fat $<50 \mathrm{~g}$ ) & Parallel & Mixed nuts & $75 \mathrm{~g}$ & $3 m$ \\
\hline Jenkins et al.-HD (32) & 2018 & 117 & Canada & $61(8.9)$ & $25(21 \%)$ & Low-fat control (Muffins, total fat $<50 \mathrm{~g}$ ) & Parallel & Mixed nuts & $37.5 \mathrm{~g}$ & $3 m$ \\
\hline Cohen et al. (37) & 2011 & 19 & USA & $66(3.3)$ & $14(74 \%)$ & Low-fat control (Cheese, $12 \mathrm{~g}$ fat) & Crossover & Almonds & $29 \mathrm{~g}$ & $12 \mathrm{w}$ \\
\hline Damavandi et al. (35) & 2013 & 50 & Iran & $55.7(7.7)$ & $34(68 \%)$ & Self-selected casual diet & Parallel & Hazelnuts & $29 \mathrm{~g}$ & $8 w$ \\
\hline Sweazea et al. (38) & 2014 & 24 & USA & $56.2(7.4)$ & $12(57 \%)$ & Diabetic diet & Parallel & Almond & $6-9 \mathrm{~g}$ & $12 w$ \\
\hline Wien et al. (39) & 2014 & 60 & USA & $61.5(12.6)$ & $30(50 \%)$ & $\begin{array}{l}\text { an ADA meal plan ( } 35 \% \text { total fat, } 45 \% \\
\text { carbohydrate, } 20 \% \text { protein) and tree nuts }\end{array}$ & Parallel & Peanuts & 2 oz per day $(56.7 \mathrm{~g})$ & $24 w$ \\
\hline Sauder et al. (40) & 2015 & 30 & USA & $56.1(7.8)$ & $15(50 \%)$ & $\begin{array}{l}\text { Low-fat control ( } 26.9 \% \text { total fat, } 186 \mathrm{mg} / \text { day } \\
\text { cholesterol) }\end{array}$ & Crossover & Pistachios & $59-128 \mathrm{~g}$ & $2 w$ \\
\hline Chiao-Ming et al. (34) & 2017 & 40 & Taiwan (China) & $54.9(10.5)$ & $20(61 \%)$ & $\begin{array}{l}\text { Low-fat control ( } 28 \% \text { fat, } 17 \% \text { protein, } 55 \% \\
\text { carbohydrate) }\end{array}$ & Crossover & Almonds & $60 \mathrm{~g}$ & $28 w$ \\
\hline Mohan et al. (44) & 2018 & 300 & India & $50.8(9.5)$ & 15 (52\%) & Diabetic diet & Parallel & Cashew & $30 \mathrm{~g}$ & $12 \mathrm{w}$ \\
\hline Sedaghat et al. (22) & 2019 & 70 & Iran & $50.1(8.7)$ & 38 (586\%) & Diabetic diet & Parallel & Soy nut & $60 \mathrm{~g}$ & $8 w$ \\
\hline
\end{tabular}

$H F$, high fat; $L F$, low fat; $F D$, full dose; $H D$, half dose.

*We define a daily diet with a fat ratio $>30 \%$ or an intake $>50 \mathrm{~g}$ as a high-fat control diet, and vice versa is a low-fat control diet. 


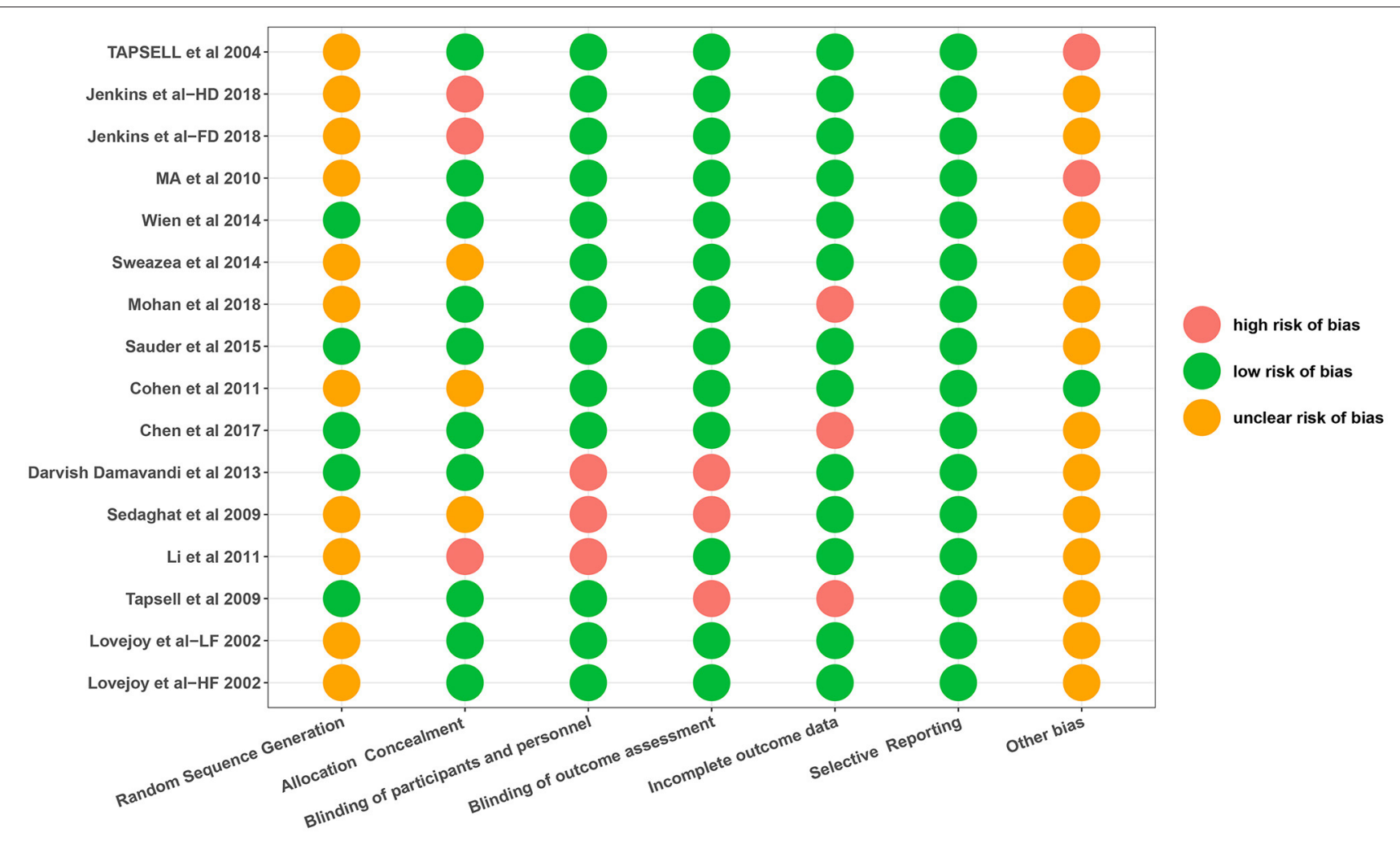

FIGURE 2 | Risk of bias assessment.

\section{Effect of Peanuts and Tree Nuts on LDL-C}

Eleven trials conducted intervention dietary trials on estimating the effects of peanuts and tree nuts consumption on LDL-C in patients with type 2 diabetes (22, 32, 33, 35-40, 44). Four studies $(22,32,33,36)$ found that tree nuts or peanuts intake significantly decreased LDL-C. However, when 11 studies were included in the meta-analysis $(n=818)$, the result revealed that consuming tree nuts or peanuts did not cause a significant reduction in LDL-C in patients with T2DM (mean difference $=-0.11 ; 95 \% \mathrm{CI}$ : $-0.25-$ $0.03, p=0.117$ ) (Figure 3). There was significant heterogeneity exhibited between studies $\left(I^{2}=53 \%, p=0.02\right)$.

\section{Effect of Peanuts and Tree Nuts on HDL-C}

Ten studies investigated the effects of supplementing peanuts and tree nuts on HDL-C in patients with type 2 diabetes $(22,32,33,35,36,38-40,44)$. Damavandi et al. (35) found that the tree nuts group significantly decreased HDL-C. Mohan et al. (44) found that participants in the nuts group have a greater increase in HDL cholesterol compared with controls. However, when the data from included trials were pooled for meta-analysis $(n=805)$, it revealed that consuming tree nuts or peanuts have no significant reduction on HDL-C in patients with $\mathrm{T} 2 \mathrm{DM}$ (mean difference $=0.01$; 95\%CI: $-0.01-0.04, p=0.400$ ) (Figure 4), and no significant between-studies heterogeneity was observed $\left(I^{2}=44 \%, p\right.$ $=0.06$ ).

\section{Effect of Peanuts and Tree Nuts on TC}

Sixteen trials explored the effects of peanuts and tree nuts supplementation on TC $(22,31-40,42-44)$. Only three studies $(22,40,42)$ found that tree nuts and peanuts significantly decreased TC. A meta-analysis included 16 studies $(n=$ 1,074 ) indicated that peanuts and tree nuts consumption can significantly reduce TC concentration in patients with T2DM (mean difference $=-0.14$; 95\%CI: $-0.26--0.02, P=$ 0.024) (Figure 5). We observed a significant between-studies heterogeneity $\left(I^{2}=45 \%, p=0.03\right)$.

\section{Effect of Peanuts and Tree Nuts on TG}

Thirteen studies appraised the effects of peanuts and tree nuts on TG $(22,32-38,40,42-44)$. Only two trials $(22,40)$ found that the tree nuts group significantly decreased TG. Moreover, when the data from included trials $(n=897)$ was pooled for meta-analysis, it revealed that peanuts and tree nuts intake can significantly lower TG concentration in patients with T2DM (mean difference $=-0.10$; 95\%CI: $-0.17--0.02, p=0.010$ ) (Figure 6), and we found no significant heterogeneity between studies $\left(I^{2}=0 \%, p\right.$ $=0.53)$.

\section{Subgroup Analysis}

The effects of peanuts and tree nuts intake on LDL-C and TC in subgroups based on characteristics of included studies are presented in Supplementary Data 7. For TC, the results 


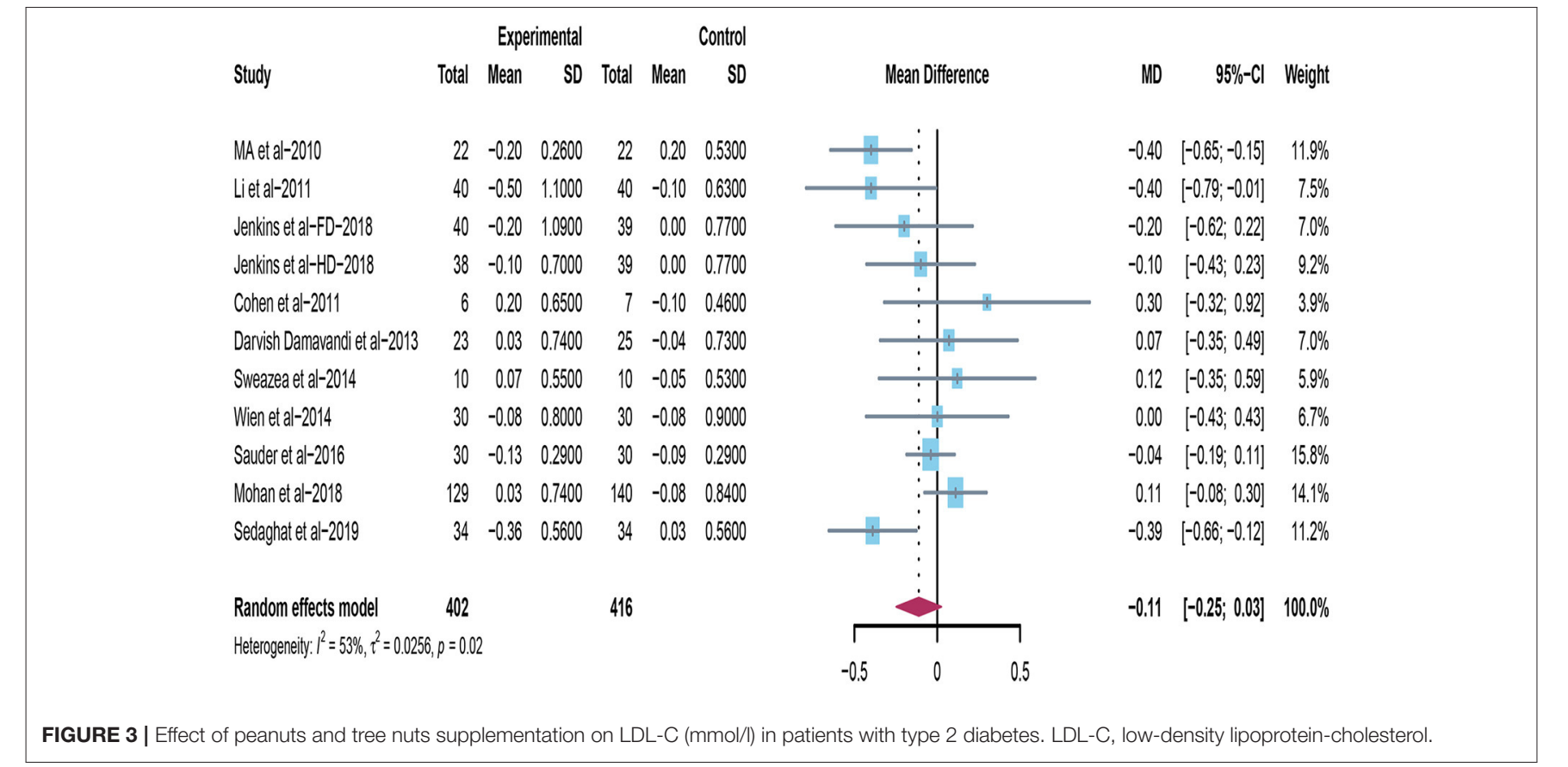

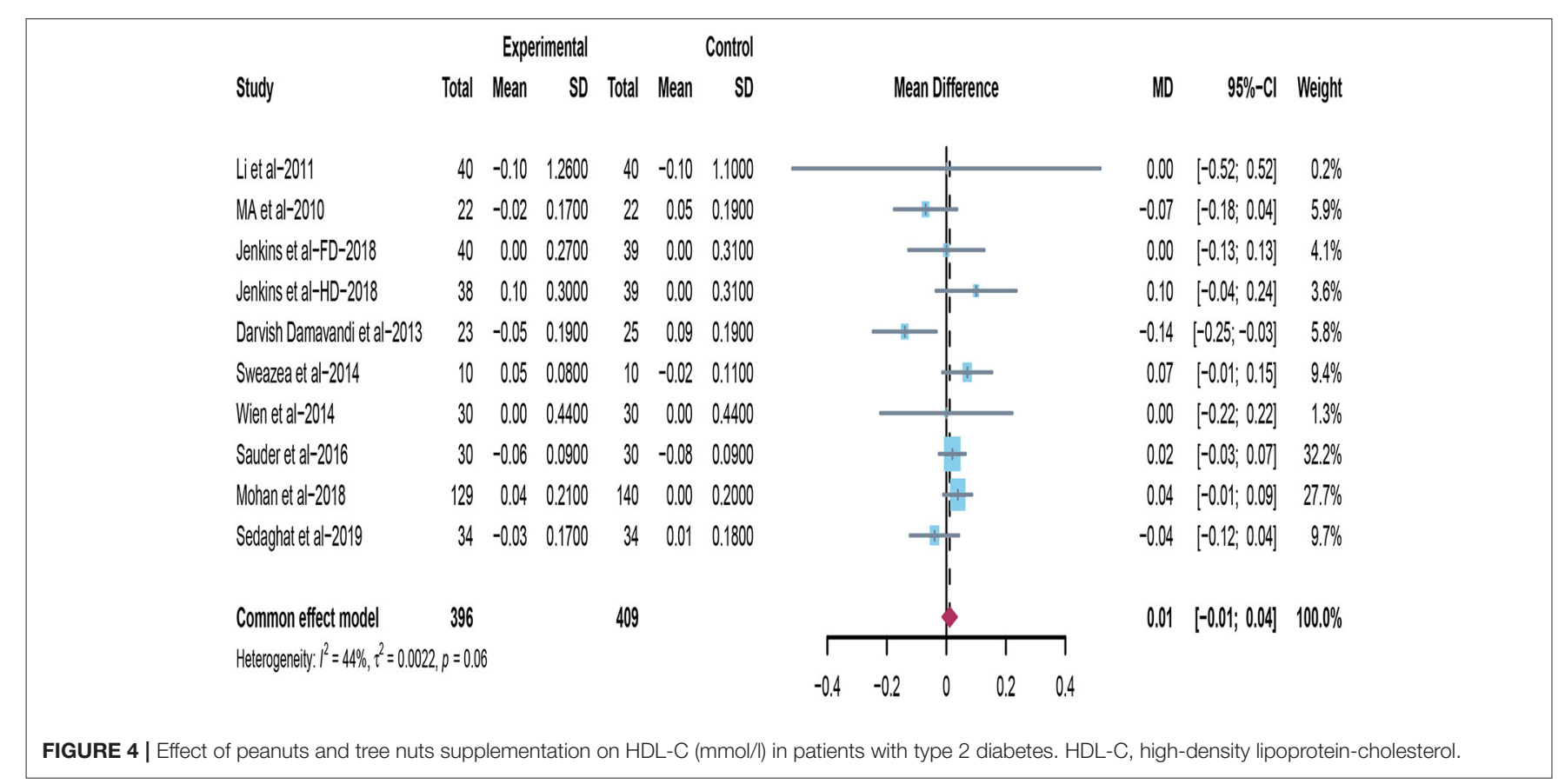

suggested that the duration of trials might be the heterogeneity source, and a significantly greater reduction in TC concentration was observed in studies whose duration was $<12$ weeks. However, we did not observe significant differences in effects by nut type, given that relatively few trials of certain nut types were available. For LDL-C, we did not find the sources of significant heterogeneity in the subgroup analysis. Furthermore, we conducted the meta-regression but did not find the relevant sources of significant heterogeneity.

\section{Sensitivity Analysis}

We performed a sensitivity analysis to evaluate the stability of our present results. The overall results generally were robust to the use of different derived correlation coefficients (Supplementary Data 4). Also, no significant changes were observed when we used the random-effects model with HartungKnapp-Sidik-Jonkman adjustment (Supplementary Data 5). In addition, sensitivity analysis showed that the removal of any of the studies from our study did not considerably change the effect 


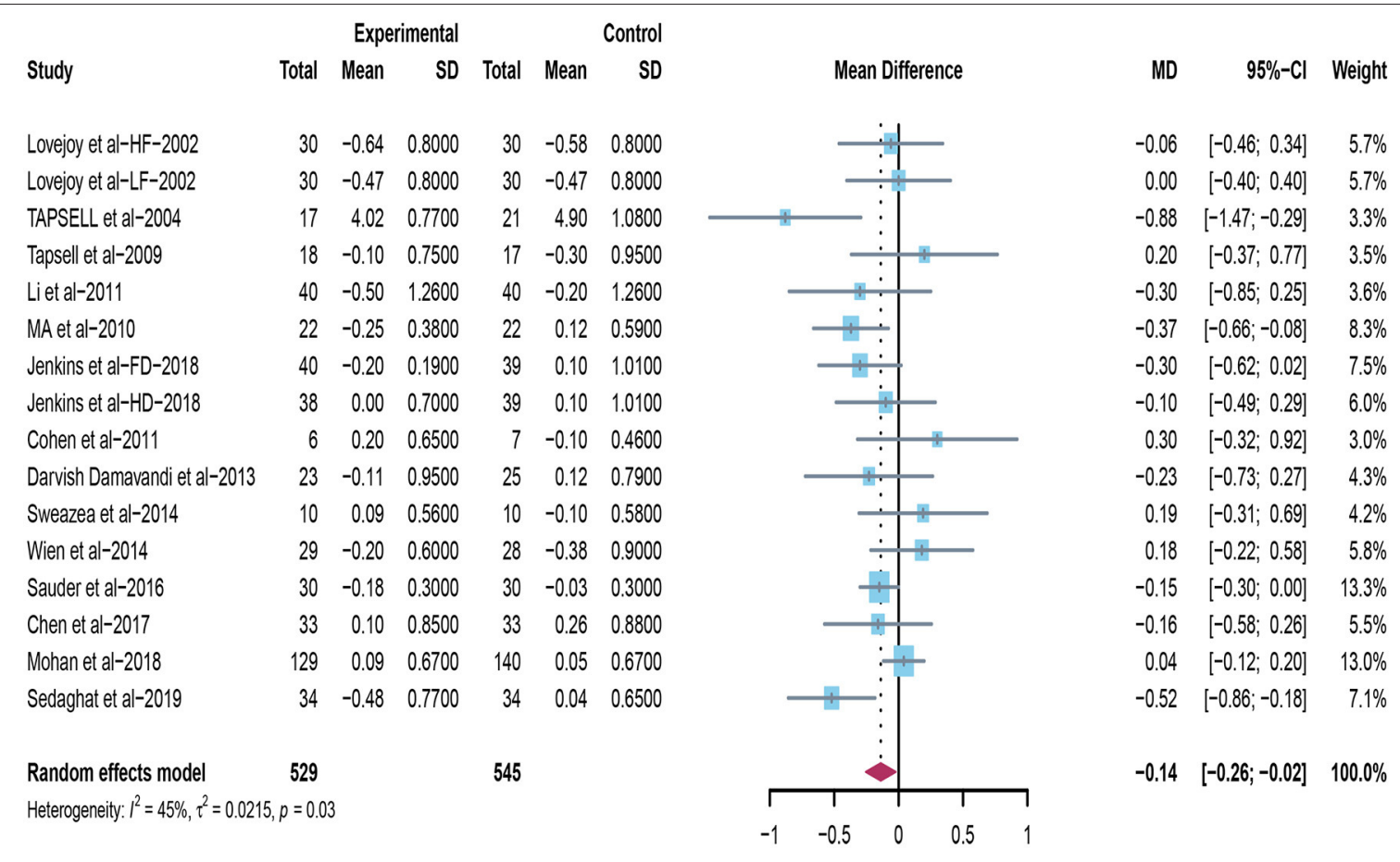

FIGURE 5 | Effect of peanuts and tree nuts supplementation on total cholesterol (mmol/l) in patients with type 2 diabetes.

of peanuts and tree nuts consumption on LDL-C, HDL-C, TC, and TG (Supplementary Data 6). These results further suggest that our results are reliable and robust.

\section{Publication Bias Regarding Peanuts and Tree Nuts' Effect on Lipid Profile}

We assessed potential publication biases by using Funnel plots, Begg's rank correlation test, Egger's regression test. Visual scanning of the funnel plot of LDL-C, HDL-C, TC, and TG suggested no asymmetry. These observations were confirmed by LDL-C Egger's regression test (intercept $=-0.22$; standard error $=1.12 ; 95 \% \mathrm{CI}:-2.76-2.32 ; t=-0.19$; two-tailed $p$ value $=0.851)$, HDL-C Egger's regression test (intercept $=-0.68$; standard error $=0.88$; 95\% CI: $-2.74-1.34 ; t=-0.78$; twotailed $p$-value $=0.460$ ), TC Egger's regression test (intercept $=-0.34$; standard error $=0.77 ; 95 \% \mathrm{CI}:-1.99-1.32 ; t=$ -0.44 ; two-tailed $p$-value $=0.670$ ), and TG Egger's regression test (intercept $=-0.08$; standard error $=0.53$; 95\% CI: $-1.25-1.09 ; t$ $=-0.15$; two-tailed $p$-value $=0.884)$. Meanwhile, results did not indicate evidence of publication bias, which were confirmed by LDL-C Begg's rank correlation test ( $z$ with continuity corrected $=1.25$; two-tailed $p$-value with continuity corrected $=0.213$ ), HDL-C Begg's rank correlation test ( $z$ with continuity corrected $=0$; two-tailed $p$-value with continuity corrected $=1.000)$, TC Begg's rank correlation test $(z$ with continuity corrected $=0.27$; two-tailed $P$-value with continuity corrected $=0.787)$, and TG Begg's rank correlation test $(z$ with continuity corrected $=0.67$; two-tailed $p$-value with continuity corrected $=0.502)($ Figure 7$)$.

\section{The Quality of the Body of Evidence}

The quality of evidence was determined using the GRADE system (Supplementary Data 8). The quality of evidence was "moderate" for TC and TG, after being downgraded due to inconsistency. The quality of evidence for HDL-C was "low," as a result of being downgraded for both inconsistency and imprecision. The quality of the body of evidence for LDL-C was "low," as a result of being downgraded for both risk of bias and inconsistency.

\section{DISCUSSION}

To the best of our knowledge, dyslipidemia is associated with the development of type 2 diabetes, and few meta-analyses were found to comprehensively analyze pieces of evidence from randomized controlled trials on the efficacy of peanuts and tree nuts on lipids profile in patients diagnosed with T2DM. The novelty of our study is to explore the effects of peanuts and tree nuts consumption on lipid profile in type 2 diabetes population based on a certain amount of randomized controlledfeeding dietary trials. The results of our meta-analysis did not reveal any significant changes in LDL-C and HDL-C following supplementation with peanuts and tree nuts in patients with type 2 diabetes. Meanwhile, our results indicated a significant reduction of TC concentration (mean difference $=-0.14$; 95\%CI: $-0.26--0.02, p=0.024)$ and TG concentration (mean difference $=-0.10 ; 95 \% \mathrm{CI}:-0.17--0.02, p=0.010$ ) following consuming peanuts and tree nuts in patients with T2DM. For TC, subgroup analysis revealed that the duration of 


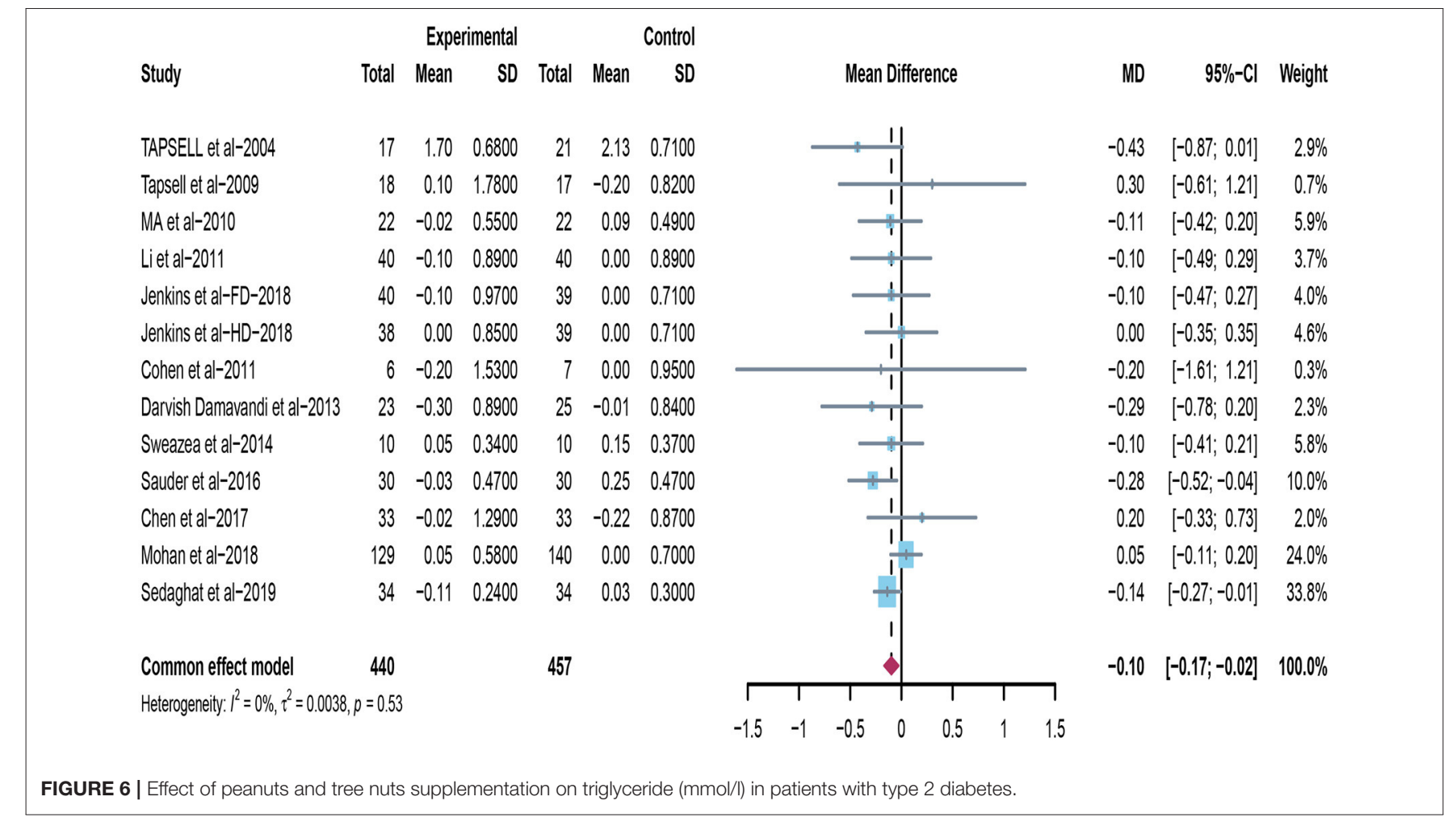

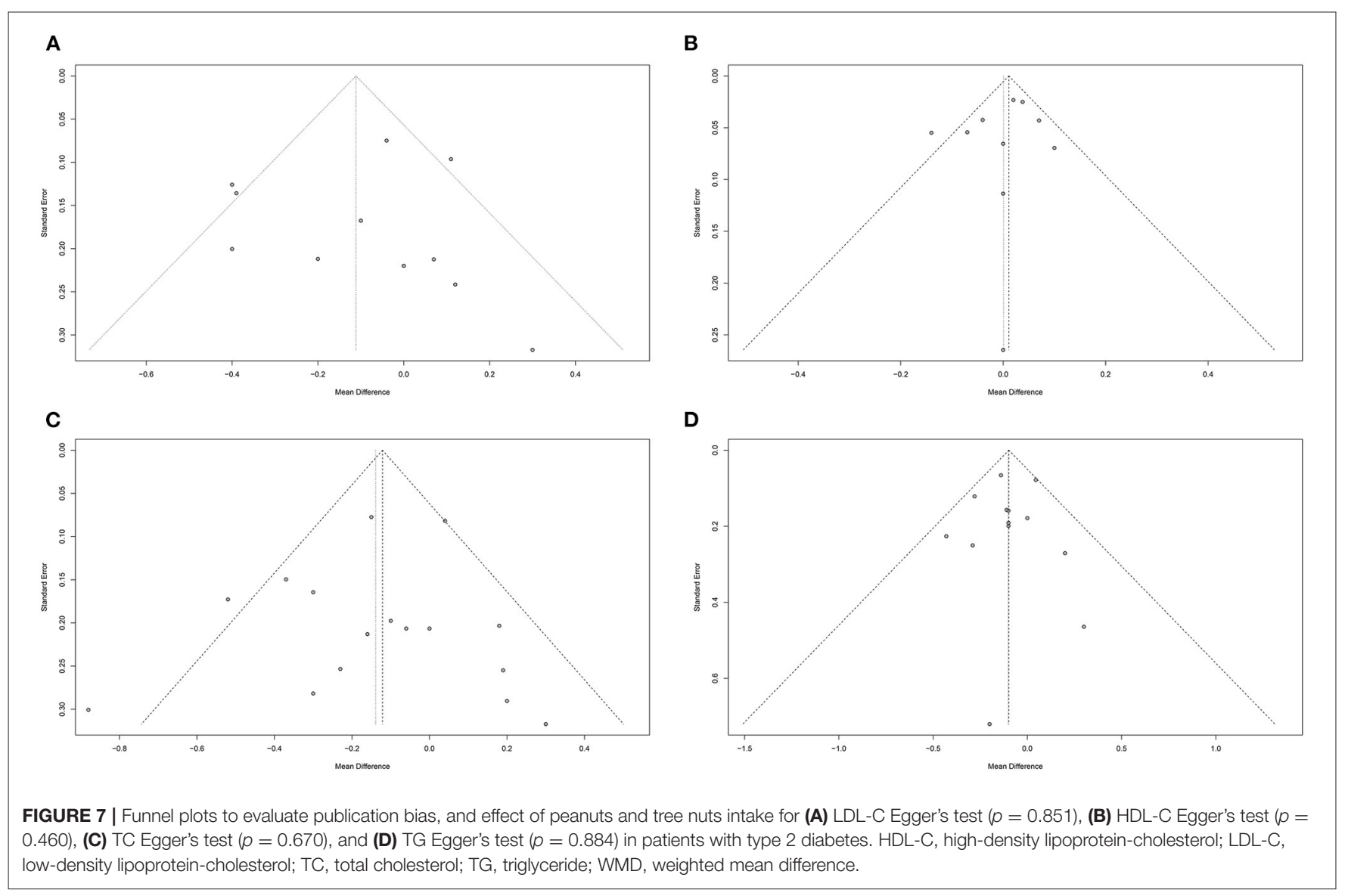


trials might contribute to the heterogeneity source, and we found a significantly greater reduction in TC concentration among studies whose duration was $<12$ weeks. However, we did not observe significant differences in effects when the type of nut is limited. Furthermore, we did not find the sources of significant heterogeneity in the subgroup analysis for LDL-C. Obvious heterogeneity still existed in some subgroups, and we failed to explain the heterogeneity completely. In sensitivity analysis, the pooled estimates were relatively robust for LDL-C, HDL-C, TC, and TG.

Most outcomes concluded from our meta-analysis were similar to previous relevant studies. Blanco Mejia et al. (17) reported a significant decrease of TGs through consuming tree nuts in the overall analysis. A TG-reducing effect had also been observed in individual trials and meta-analysis of trials appraising the benefit of the Mediterranean diet in patients with T2DM (46). It was reported that the TG-decreasing effect was followed by an increased HDL-C (46). However, the present results revealed that peanuts and tree nuts did not significantly increase HDL-C concentration in patients with T2DM. It has been well-known that tree nuts contain many compounds such as polyunsaturated fatty acid, monounsaturated fatty acid, and fiber. To some extent, the benefits of tree nuts for improving blood lipid might be attributed to various kinds of nutrients which contribute to cardiovascular protection, such as unsaturated fatty acids, fiber, and phytochemicals (32). The fiber content and high unsaturated fat from tree nuts can substitute high glycemic index carbohydrates from the diet, thereby causing a reduction of the glycemic load in the diet, which may be the main facilitating factor in lowering TG (47).

Del Gobbo et al. (16) reported a significant decrease in TC and LDL cholesterol through tree nuts consumption. We also observed a similar significant reduction in TC. The first clinical trial in 1993 exploring the effects of walnuts in healthy men concluded that a walnuts diet can significantly decrease TC and LDL-C concentration (21). In addition, some wellcontrolled intervention trials with tree nuts also confirmed that tree nuts have cholesterol-lowering efficacy (48-52). When the population was limited to people with T2DM, our results showed that tree nuts and peanuts can significantly lower cholesterol concentration, which was consistent with the results of previous studies focused on healthy or hyperlipidemia patients. Nuts are rich in phytosterols, which may promote their TC-lowering effect (53).

Heterogeneity for TC may be explained by the duration of trials. We found a significantly greater reduction of TC concentration in studies whose duration was $<12$ weeks, it may be that the length of the intervention has a direct impact on the effect, and the longer intervention period and the shorter intervention period may have opposite outcomes. Also, given the relatively few studies for certain nut types, we did not observe significant differences in effects by nut type. In addition, we could not explore the possible source of heterogeneity for LDL-C. We attempted to explain heterogeneity by performing subgroup and sensitivity analyses, however, for the most part, heterogeneity remained. Also, no publication bias was detected for LDL-C, HDL-C, TC, and TG.

Peanuts and tree nuts, as a kind of nutritious food in daily life, are easy to obtain for subjects with type 2 diabetes. The management of blood lipids in patients with type 2 diabetic is also as important as blood sugar control and needs to be taken seriously. Our meta-analysis results revealed that daily intake of peanuts and tree nuts may help control TC and TGs. In addition, our results did not find that the intake of peanuts and tree nuts can lower HDL-C and LDL-C. Thus, peanut and tree nuts consumption can be considered as a healthy lifestyle to help manage the blood lipids in patients with type 2 diabetes.

This meta-analysis has several strengths. Our study examined the efficacy of tree nuts or peanuts intake in patients with type 2 diabetes. In addition, it was performed and reported based on current guidelines $(25,54,55)$, and comprised an evaluation of results employing numerous sensitivity analyses, and investigation of the risk of bias using an updated assessment tool. There were potential limitations in our review. First, we did not prospectively register our systematic review and meta-analysis, so it may lack transparency and increase the potential for bias. Second, the majority of the trials used tree nuts or peanuts consumption as the dietary intervention at baseline. However, during the follow-up period, some participants may hide the fact that they have changed their dietary habits. Third, although random- or fixed-effects model selection based on the $I^{2}$ metrics is commonly performed, preferably the model selection should be a priority. Whether the results from present studies are expected to produce one similar result across studies is unknown in advance although we have conducted a sensitivity analysis, which could be a limitation in our study. Fourth, we could not explore the effects of nut type because of the relatively few studies for certain nut types.

\section{CONCLUSION}

In summary, evidence from our pooled analyses indicated that daily peanuts and tree nuts consumption may significantly reduce TC and TG concentration in patients with type 2 diabetes. In addition, no obvious evidence of an effect of daily peanuts and tree nuts supplementation on LDL-C concentration and HDL-C concentration was found. These findings support daily peanuts and tree nut intake, as an important part of healthy lifestyles, could be recommended in the management of blood lipids in type 2 diabetics subjects. Also, several larger-scale, high-quality randomized controlled trials of certain nut types are needed to validate the findings.

\section{DATA AVAILABILITY STATEMENT}

The datasets presented in this study can be found in online repositories. The names of the repository/repositories and accession number(s) can be found in the article/Supplementary Material. 


\section{AUTHOR CONTRIBUTIONS}

J-yX and J-hY: conceptualization. D-fX and HX: data curation and software. J-yX, D-fX, and HX: formal analysis. J-yX and CY: methodology. J-yX and G-jS: supervision and validation. HX and G-jS: visualization. J-yX: writingoriginal draft. CY and G-jS: writing-review and editing. All authors contributed to the article and approved the submitted version.

\section{REFERENCES}

1. Kris-Etherton PM, Harris WS, Appel LJ. Fish consumption, fish oil, omega3 fatty acids, and cardiovascular disease. Circulation. (2002) 106:274757. doi: 10.1161/01.CIR.0000038493.65177.94

2. Unnikrishnan $\mathrm{R}$, Pradeepa R, Joshi SR, Mohan V. Type 2 diabetes: demystifying the global epidemic. Diabetes. (2017) 66:1432. doi: $10.2337 / \mathrm{db} 16-0766$

3. Feng W, Zhao T, Mao G, Wang W, Yun F, Fang L, et al. Type 2 diabetic rats on diet supplemented with chromium malate show improved glycometabolism, glycometabolism-related enzyme levels and lipid metabolism. PLoS ONE. (2015) 10:e0125952. doi: 10.1371/journal.pone.0125952

4. Heisler M, Vijan S, Anderson RM, Ubel PA, Bernstein SJ, Hofer TP. When do patients and their physicians agree on diabetes treatment goals and strategies, and what difference does it make? J Gen Intern Med. (2003) 18:893-902. doi: 10.1046/j.1525-1497.2003.21132.x

5. Zulman DM, Kerr EA, Hofer TP, Heisler M, Zikmund-Fisher BJ. Patient-provider concordance in the prioritization of health conditions among hypertensive diabetes patients. J Gen Intern Med. (2010) 25:40814. doi: 10.1007/s11606-009-1232-1

6. Kelsey MM, Geffner ME, Guandalini C, Pyle L, White NH. Presentation and effectiveness of early treatment of type 2 diabetes in youth: lessons from the TODAY study. Pediatric Diabetes. (2016) 17:12264. doi: 10.1111/pedi.12264

7. Ros E. Health benefits of nut consumption. Nutrients. (2010) 2:65282. doi: 10.3390/nu2070652

8. Brufau G, Boatella J, Rafecas M. Nuts: source of energy and macronutrients. Br J Nutr. (2006) 96(Suppl.2):S24. doi: 10.1017/BJN20061860

9. Ros E, Mataix J. Fatty acid composition of nutsimplications for cardiovascular health. $\mathrm{Br} J$ Nutr. (2006) 96(Suppl.2):S29. doi: 10.1017/BJN20061861

10. D'Alessandro A, De Pergola G. Mediterranean diet pyramid: a proposal for Italian people. Nutrients. (2014) 6:4302-16. doi: 10.3390/nu6104302

11. Fogacci F, Cicero AFG, Derosa G, Rizzo M, Veronesi M, Borghi C. Effect of pistachio on brachial artery diameter and flow-mediated dilatation: a systematic review and meta-analysis of randomized, controlled-feeding clinical studies. Crit Rev Food Sci Nutr. (2019) 59:328-35. doi: 10.1080/10408398.2017.1368007

12. Gervasi T, Barreca D, Laganà G, Mandalari G. Health benefits related to tree nut consumption and their bioactive compounds. Int J Mol Sci. (2021) 22:115960. doi: 10.3390/ijms22115960

13. Nikodijevic CJ, Probst YC, Batterham MJ, Tapsell LC, Neale EP. Nut consumption in a representative survey of Australians: a secondary analysis of the 2011-2012 National Nutrition and Physical Activity Survey. Public Health Nutr. (2020) 23:3368-78. doi: 10.1017/S1368980019004117

14. Núria I, Mònica B, Marta GF, Emilio R, Martínez-González M, Dolores C, et al. Cross-sectional assessment of nut consumption and obesity, metabolic syndrome and other cardiometabolic risk factors: the PREDIMED study. PLoS ONE. (2013) 8:e57367. doi: 10.1371/journal.pone.0057367

15. Banel DK, Hu FB. Effects of walnut consumption on blood lipids and other cardiovascular risk factors: a meta-analysis and systematic review. Am J Clin Nutr. (2009) 1:56-63. doi: 10.3945/ajcn.2009.27457

16. Del GLC, Falk MC, Robin F, Kara L, Dariush M. Effects of tree nuts on blood lipids, apolipoproteins, and blood pressure: systematic review, meta-analysis,

\section{ACKNOWLEDGMENTS}

The authors sincerely thank the support from each participant.

\section{SUPPLEMENTARY MATERIAL}

The Supplementary Material for this article can be found online at: https://www.frontiersin.org/articles/10.3389/fnut.2021. 765571/full\#supplementary-material

and dose-response of 61 controlled intervention trials. Am J Clin Nutr. (2015) 6:1347-56. doi: 10.3945/ajcn.115.110965

17. Mejia SB, Kendall C, Viguiliouk E, Augustin LS, Ha V, Cozma AI, et al. Effect of tree nuts on metabolic syndrome criteria: a systematic review and meta-analysis of randomised controlled trials. BMJ Open. (2013) 4:e004660. doi: 10.1136/bmjopen-2013-004660

18. Jenkins DJA, Kendall CWC, Marchie A, Parker TL, Connelly PW, Qian W, et al. Dose response of almonds on coronary heart disease risk factors: blood lipids, oxidized low-density lipoproteins, lipoprotein(a), homocysteine, and pulmonary nitric oxide: a randomized, controlled, crossover trial. Circulation. (2002) 106:1327-32. doi: 10.1161/01.CIR.0000028421.91733.20

19. Ros E, Núñez I, Pérez-Heras A, Serra M, Gilabert R, Casals E, et al. walnut diet improves endothelial function in hypercholesterolemic subjects: a randomized crossover trial. Circulation. (2004) 109:160914. doi: 10.1161/01.CIR.0000124477.91474.FF

20. Iwamoto M, Imaizumi K, Sato M, Hirooka Y, Sakai K, Takeshita A, et al. Serum lipid profiles in Japanese women and men during consumption of walnuts. Eur J Clin Nutr. (2002) 56:629-37. doi: 10.1038/sj.ejcn.1601400

21. Sabaté J, Fraser GE, Burke K, Knutsen SF, Bennett H, Lindsted KD. Effects of walnuts on serum lipid levels and blood pressure in normal men. $N$ Engl J Med. (1993) 328:603-7. doi: 10.1056/NEJM199303043280902

22. Sedaghat A, Shahbazian H, Rezazadeh A, Haidari F, Jahanshahi A, Mahmoud Latifi S, et al. The effect of soy nut on serum total antioxidant, endothelial function and cardiovascular risk factors in patients with type 2 diabetes. Diabetes Metab Syndr. (2019) 13:1387-91. doi: 10.1016/j.dsx.2019.01.057

23. Bodnaruc AM, Prud'homme D, Giroux I. Acute effects of an isocaloric macronutrient-matched breakfast meal containing almonds on glycemic, hormonal, and appetite responses in men with type 2 diabetes: a randomized crossover study. Appl Physiol Nutr Metabol. (2020) 45:5209. doi: 10.1139/apnm-2019-0559

24. Hou YY, Ojo O, Wang LL, Wang Q, Jiang Q, Shao XY, et al. A randomized controlled trial to compare the effect of peanuts and almonds on the cardiometabolic and inflammatory parameters in patients with type 2 diabetes mellitus. Nutrients. (2018) 10:111565. doi: 10.3390/nu10111565

25. Page MJ, McKenzie JE, Bossuyt PM, Boutron I, Hoffmann TC, Mulrow CD, et al. The PRISMA 2020 statement: an updated guideline for reporting systematic reviews. BMJ. (2021) 372:n71. doi: 10.1136/bmj.n71

26. Wittekind A, Higgins K, McGale L, Schwartz C, Stamataki NS, Beauchamp GK, et al. A workshop on 'Dietary Sweetness-Is It an Issue?' Int J Obes. (2018) 42:934-8. doi: 10.1038/ijo.2017.296

27. Higgins JPT, Altman DG, Gøtzsche PC, Jüni P, Moher D, Oxman AD, et al. The Cochrane Collaboration's tool for assessing risk of bias in randomised trials. BMJ. (2011) 343:d5928. doi: 10.1136/bmj.d5928

28. Feldman EB. The scientific evidence for a beneficial health relationship between walnuts and coronary heart disease. J Nutr. (2002) 132:1062S. doi: 10.1093/jn/132.5.1062S

29. Duval S, Tweedie R. Trim and fill: a simple funnel-plot-based method of testing and adjusting for publication bias in meta-analysis. Biometrics. (2000) 56:455-63. doi: 10.1111/j.0006-341X.2000.00455.x

30. Guyatt GH, Oxman AD, Vist GE, Kunz R, Falck-Ytter Y, Alonso-Coello $\mathrm{P}$, et al. an emerging consensus on rating quality of evidence and strength of recommendations. BMJ. (2008) 336:924-6. doi: 10.1136/bmj.39489.470347.AD 
31. Lovejoy JC, Most MM, Lefevre M, Greenway FL, Rood JC. Effect of diets enriched in almonds on insulin action and serum lipids in adults with normal glucose tolerance or type 2 diabetes. Am J Clin Nutr. (2002) 76:10006. doi: 10.1093/ajcn/76.5.1000

32. Jenkins DJA, Kendall CWC, Lamarche B, Banach MS, Srichaikul K, Vidgen E, et al. Nuts as a replacement for carbohydrates in the diabetic diet: a reanalysis of a randomised controlled trial. Diabetologia. (2018) 61:173447. doi: 10.1007/s00125-018-4628-9

33. Li SC, Liu YH, Liu JF, Chang WH, Chen CM, Chen CYO. Almond consumption improved glycemic control and lipid profiles in patients with type 2 diabetes mellitus. Metabolism. (2011) 60:474-9. doi: 10.1016/j.metabol.2010.04.009

34. Chiao-Ming C, Jen-Fang L, Sing-Chung L, Chen-Ling H, An-Tsz H, ShuenFu W, et al. Almonds ameliorate glycemic control in Chinese patients with better controlled type 2 diabetes: a randomized, crossover, controlled feeding trial. Nutr Metabol. (2017) 14:1-12. doi: 10.1186/s12986-0170205-3

35. Damavandi RD, Eghtesadi S, Shidfar F, Heydari I, Foroushani AR. Effects of hazelnuts consumption on fasting blood sugar and lipoproteins in patients with type 2 diabetes. J Res Med Sci. (2013) 18:314-21.

36. Ma Y, Njike VY, Millet J, Dutta S, Doughty K, Treu JA, et al. Effects of walnut consumption on endothelial function in type 2 diabetic subjects: a randomized controlled crossover trial. Diabetes Care. (2010) 33:22732. doi: $10.2337 / \mathrm{dc} 09-1156$

37. Cohen AE, Johnston CS. Almond ingestion at mealtime reduces postprandial glycemia and chronic ingestion reduces hemoglobin $\mathrm{A}(1 \mathrm{c})$ in individuals with well-controlled type 2 diabetes mellitus. Metabolism. (2011) 60:13127. doi: 10.1016/j.metabol.2011.01.017

38. Sweazea KL, Johnston CS, Ricklefs KD, Petersen KN. Almond supplementation in the absence of dietary advice significantly reduces C-reactive protein in subjects with type 2 diabetes. J Funct Foods. (2014) 10:252-9. doi: 10.1016/j.jff.2014.06.024

39. Wien M, Oda K, Sabaté J. A randomized controlled trial to evaluate the effect of incorporating peanuts into an American Diabetes Association meal plan on the nutrient profile of the total diet and cardiometabolic parameters of adults with type 2 diabetes. Nutr J. (2014) 13:10. doi: 10.1186/1475-2891-13-10

40. Sauder KA, McCrea CE, Ulbrecht JS, Kris-Etherton PM, West SG. Effects of pistachios on the lipid/lipoprotein profile, glycemic control, inflammation, and endothelial function in type 2 diabetes: a randomized trial. Metabolism. (2015) 64:1521-9. doi: 10.1016/j.metabol.2015. 07.021

41. Jenkins DJA, Kendall CWC, Banach MS, Srichaikul K, Vidgen E, Mitchell S, et al. Nuts as a replacement for carbohydrates in the diabetic diet. Diabetes Care. (2011) 34:1706-11. doi: 10.2337/dc11-0338

42. Tapsell LC, Gillen LJ, Patch CS, Batterham M, Owen A, Bare M, et al. Including walnuts in a low-fat/modified-faf diet improves HDL cholesterolto-total cholesterol ratios in patients with type 2 diabetes. Diabetes Care. (2004) 27:2777-83. doi: 10.2337/diacare.27.12.2777

43. Tapsell LC, Batterham MJ, Teuss G, Tan SY, Dalton S, Quick CJ, et al. Long-term effects of increased dietary polyunsaturated fat from walnuts on metabolic parameters in type II diabetes. Eur J Clin Nutr. (2009) 63:100815. doi: 10.1038/ejcn.2009.19

44. Mohan V, Gayathri R, Jaacks LM, Lakshmipriya N, Anjana RM, Spiegelman D, et al. Cashew nut consumption increases HDL cholesterol and reduces systolic blood pressure in asian indians with type 2 diabetes: a 12-week randomized controlled trial. J Nutr. (2018) 148:63-9. doi: 10.1093/jn/nxx001

45. Sauder KA, McCrea CE, Ulbrecht JS, Kris-Etherton PM, West SG. Pistachio nut consumption modifies systemic hemodynamics, increases heart rate variability, and reduces ambulatory blood pressure in wellcontrolled type 2 diabetes: a randomized trial. J Am Heart Assoc. (2014) 3:873. doi: 10.1161/JAHA.114.000873

46. Esposito K, Maiorino MI, Ciotola M, Di Palo C, Scognamiglio P, Gicchino $M$, et al. Effects of a Mediterranean-style diet on the need for antihyperglycemic drug therapy in patients with newly diagnosed type 2 diabetes: a randomized trial. Ann Intern Med. (2009) 151:30614. doi: 10.7326/0003-4819-151-5-200909010-00004

47. Kris-Etherton PM, Hu FB, Ros E, Sabaté J. The role of tree nut and peanuts in the prevention of coronary heart disease: multiple potential mechanisms. J Nutr. (2008) 138:1746S-51S. doi: 10.1093/jn/138.9. $1746 \mathrm{~S}$

48. Rajaram S, Haddad EH, Mejia A, Sabate J. Walnuts and fatty fish influence different serum lipid fractions in normal to mildly hyperlipidemic individuals: a randomized controlled study. Am J Clin Nutr. (2009) 89:1657S. doi: 10.3945/ajcn.2009.26736S

49. Torabian-Riasati S. Long-term walnut supplementation without dietary advice induces favorable serum lipid changes in free-living individuals. Eur J Clin Nutr. (2010) 65:274-9. doi: 10.1038/ejcn.2009.152

50. Jenkins D, Kendall C, Augustine M, Josse AR, Nguyen TH, Faulkner DA, et al. Almonds reduce biomarkers of lipid peroxidation in older hyperlipidemic subjects. J Nutr. (2008) 138:908-13. doi: 10.1093/jn/138.5.908

51. Griel AE, Cao Y, Bagshaw DD, Cifelli AM, Kris-Etherton PM. A macadamia nut-rich diet reduces total and LDL-cholesterol in mildly hypercholesterolemic men and women. J Nutr. (2008) 138:761-7. doi: 10.1093/jn/138.4.761

52. Lokko P, Lartey A, Armar-Klemesu M, Mattes RD. Regular peanut consumption improves plasma lipid levels in healthy Ghanaians. Int J Food Sci Nutr. (2007) 58:190-200. doi: 10.1080/09637480701198067

53. Segura R, Javierre C, Lizarraga MA, Ros E. Other relevant components of nuts: phytosterols, folate and minerals. Br J Nutr. (2006) 96:S36. doi: 10.1017/BJN20061862

54. Cumpston M, Li T, Page MJ, Chandler J, Welch VA, Higgins JP, et al. Updated guidance for trusted systematic reviews: a new edition of the Cochrane Handbook for Systematic Reviews of Interventions. Cochr Database Systemat Rev. (2019) 10:ED000142. doi: 10.1002/14651858.ED000142

55. Boon $\mathrm{MH}$, Thomson $\mathrm{H}$. The effect direction plot revisited: application of the 2019 Cochrane Handbook guidance on alternative synthesis methods. Res Synth Methods. (2021) 12:29-33. doi: 10.1002/jrsm.1458

Conflict of Interest: The authors declare that the research was conducted in the absence of any commercial or financial relationships that could be construed as a potential conflict of interest.

Publisher's Note: All claims expressed in this article are solely those of the authors and do not necessarily represent those of their affiliated organizations, or those of the publisher, the editors and the reviewers. Any product that may be evaluated in this article, or claim that may be made by its manufacturer, is not guaranteed or endorsed by the publisher.

Copyright (c) $2021 \mathrm{Xia}, \mathrm{Yu}, \mathrm{Xu}$, Yang, Xia and Sun. This is an open-access article distributed under the terms of the Creative Commons Attribution License (CC BY). The use, distribution or reproduction in other forums is permitted, provided the original author(s) and the copyright owner(s) are credited and that the original publication in this journal is cited, in accordance with accepted academic practice. No use, distribution or reproduction is permitted which does not comply with these terms. 